\title{
Automorphisms of elliptic K3 surfaces and Salem numbers of maximal degree
}

\author{
Hélène Esnault, Keiji Oguiso and Xun Yu \\ Dedicated to Professor Shing-Tung Yau on the occasion of his sixty-fifth birthday
}

\begin{abstract}
Using elliptic structures, we show that any supersingular K3 surface of Artin invariant 1 in characteristic $p \neq 5,7,13$ has an automorphism the entropy of which is the natural logarithm of a Salem number of degree 22 .
\end{abstract}

\section{Introduction}

If $p$ is a prime number, there is an Artin invariant 1 supersingular K3 surface $X(p)$, defined over the congruence field $\mathbb{F}_{p}$. It is unique up to isomorphism (see Section 2). Since Ogus' seminal work [Ogu79, Ogu83], this surface has been studied from various viewpoints.

In [ES13] it is shown that, as in characteristic 0, in positive characteristic the maximum of the absolute values of the algebraic integers which are eigenvalues of an automorphism of a smooth projective surface acting on its $\ell$-adic cohomology is taken on the Néron-Severi group. Thus by analogy with complex geometry, the logarithm of this maximum is called the entropy. One knows that the entropy of an automorphism of a K3 surface is either 0 or the logarithm of a Salem number (see Section 3), hence of degree at most the rank of the Néron-Severi group, thus at most 20 for projective K3 surfaces in characteristic 0 .

Over $k=\overline{\mathbb{F}}_{p}$, Jang [Jan14] showed that the image of the canonical representation

$$
\operatorname{Aut}(X(p)) \rightarrow \operatorname{GL}\left(H^{0}\left(X(p), \omega_{X(p)}\right)\right) \simeq k^{\times}
$$

is isomorphic to the cyclic group of order $p+1$. In particular, for $p$ large, there are automorphims which are not geometrically liftable to characteristic 0. Jang's proof relies on Ogus' Torelli theorem [Ogu83]. From this, and from Shioda's study of Mordell-Weil lattices [Shi90], it is deduced in [EO15] that for $p$ very large, there are automorphisms of $X(p)$ of positive entropy which are not geometrically liftable to characteristic 0 (see Section 2.3 for the definition of this liftability notion).

The main result of this note is the following.

Received 17 November 2014, accepted in final form 5 January 2016.

2010 Mathematics Subject Classification 14J28, 14J50, 14G20, 14 G99

Keywords: K3 surfaces, automorphisms, mixed characteristics, Salem numbers

This journal is (C) Foundation Compositio Mathematica 2016. This article is distributed with Open Access under the terms of the Creative Commons Attribution Non-Commercial License, which permits non-commercial reuse, distribution, and reproduction in any medium, provided that the original work is properly cited. For commercial re-use, please contact the Foundation Compositio Mathematica.

The first author is supported by the Einstein program and the ERC Advanced Grant 226257. The second author is supported by JSPS Grant-in-Aid (S) No 25220701, JSPS Grant-in-Aid (S) No 22224001, JSPS Grant-in-Aid (B) No 22340009, and by KIAS Scholar Program. 
TheOREM 1.1. Let $p \neq 5,7,13$. Then there is an automorphism $f \in \operatorname{Aut}(X(p))$, defined over $\overline{\mathbb{F}}_{p}$, the entropy of which is the logarithm of a Salem number of degree 22.

In particular, those automorphisms are not geometrically liftable to characteristic 0 . The result is known over $k=\overline{\mathbb{F}}_{p}$ for $p=2$ [BC13] and $p=3$ [EO15].

While for $p=2$ the proof relies on a very detailed study of $X(2)$ in [DK03], and for $p=3$ it is computer aided and relies on the explicit study of Aut $(X(3))$ in [KS14], our proof of Theorem 1.1 is abstract and based on the theory of the Mordell-Weil group of $X(p)$ by Shioda [Shi13]. We show the following strengthening of Theorem 1.1.

Theorem 1.2. Let $k$ be an algebraically closed field of characteristic $p \geqslant 0$. Let $X$ be a K3 surface over $k$ of Picard number $\rho=2 d \geqslant 4$. Assume that $X$ has two non-isomorphic elliptic fibrations $\varphi_{1}: X \rightarrow \mathbb{P}^{1}(i=1,2)$ such that the Mordell-Weil group $\mathrm{MW}\left(\varphi_{1}\right)$ of $\varphi_{1}$ is of maximal rank, that is, $2 d-2$, and $\mathrm{MW}\left(\varphi_{2}\right)$ is of positive rank. Then $X$ has an automorphism $f$, the entropy of which is the logarithm of a Salem number of degree $2 d$.

One deduces Theorem 1.1 from Theorem 1.2 using Shioda's theorem [Shi13].

ThEOREM 1.3. Let $p=11$ or $p>13$. Then $X(p)$ admits an elliptic fibration of Mordell-Weil rank $20=22-2$.

This also explains the restriction on $p$ in Theorem 1.1. It is not unlikely that this restriction in Theorem 1.1 is unnecessary. More generally, it is likely that Theorem 1.2, Theorem 4.1 (mimicking [BC13]), and Theorem 4.6 could have a larger range of applications.

In this note, we do not address some questions of more arithmetical flavor. We know that the Néron-Severi group of $X(p)$ is defined over $\mathbb{F}_{p^{2}}$ [Sch12]. Over which field are those automorphisms of Theorem 1.1 defined? This depends on the field of definition of the Mordell-Weil groups in Theorem 1.3. Further, we know that Salem numbers of bounded degree are discrete. This raises the question whether or not the minimal Salem number of degree 22 arises as the logarithm of the entropy of an automorphism on a supersingular K3 surface (see [McM16] and references there). We also know that powers of Salem numbers are Salem numbers. Given a Salem number of degree 22 which is the power of another Salem number of degree 22, and such that its logarithm is the entropy of an automorphism $f$ on a supersingular K3 surface, when is $f$ itself the power of an automorphism? Finally, it would be interesting to relate this work to [GM02, McM02], in which the authors show that any unramified degree 22 Salem number is the logarithm of the entropy of an automorphism of a non-projective complex K3 surface.

\section{Preliminaries on K3 surfaces and liftings}

In this section, we fix the notation and recall basic facts on K3 surfaces and liftings from [EO15] and references therein.

\subsection{K3 surfaces}

Let $X$ be a K3 surface defined over an algebraically closed field $k$ of characteristic $p \geqslant 0$; that is, $X$ is a smooth projective surface defined over $k$ such that $H^{1}\left(X, \mathcal{O}_{X}\right)=0$ and the dualizing sheaf is trivial: $\omega_{X} \simeq \mathcal{O}_{X}$. We denote by $\operatorname{NS}(X)$ the Néron-Severi group of $X$. Then the Picard group $\operatorname{Pic}(X)$ is isomorphic to the Néron-Severi group $\mathrm{NS}(X)$, which is a free $\mathbb{Z}$-module of finite rank. The rank of $\mathrm{NS}(X)$ is called the Picard number of $X$ and is denoted by $\rho(X)$. It is at 


\section{H. Esnault, K. Oguiso and X. Yu}

least 1 as $X$ is assumed to be projective, and at most 22 , the second $\ell$-adic Betti number. In characteristic 0 , it is at most 20 by Hodge theory. The intersection form $(*, * *)$ on $\mathrm{NS}(X)$ is of signature $(1, \rho(X)-1)$, and $(\mathrm{NS}(X),(*, * *))$ is then an even hyperbolic lattice. The dual $\mathbb{Z}_{-}^{-}$ module $\mathrm{NS}(X)^{*}:=\operatorname{Hom}_{\mathbb{Z}}(\mathrm{NS}(X), \mathbb{Z})$ is regarded as a $\mathbb{Z}$-submodule of $\mathrm{NS}(X) \otimes \mathbb{Q}$, containing $\mathrm{NS}(X)$ through the intersection form $(*, * *)$, which is non-degenerate. The quotient module $\mathrm{NS}(X)^{*} / \mathrm{NS}(X)$ is called the discriminant group of $X$.

The surface $X$ is called supersingular if $\rho(X)=22$, the maximum possible value. (As the Tate conjecture is not yet proven for $p=2$, one should rather say Shioda supersingular in this case, but we consider only supersingular K3 surfaces in odd characteristic in this note).

Artin [Art74] proved that the discriminant group $\mathrm{NS}(X)^{*} / \mathrm{NS}(X)$ of a supersingular $\mathrm{K} 3$ surface $X$ is $p$-elementary; more precisely, as an abelian group

$$
\mathrm{NS}(X)^{*} / \mathrm{NS}(X) \simeq(\mathbb{Z} / p)^{2 \sigma(X)},
$$

where $\sigma(X)$ is an integer such that $1 \leqslant \sigma(X) \leqslant 10$. The integer $\sigma(X)$ is called the Artin invariant of $X$. Let $\sigma$ be an integer such that $1 \leqslant \sigma \leqslant 10$. Then the supersingular K3 surfaces over $k$ with $\sigma(X) \leqslant \sigma$ form a $(\sigma-1)$-dimensional family over $k$. There is, up to isomorphism, a unique Artin invariant 1 supersingular K3 surface $X(p)$ in each positive characteristic $p>0$. It is defined over $\mathbb{F}_{p}$ and its Néron-Severi group is defined over $\mathbb{F}_{p^{2}}$, not over $\mathbb{F}_{p}$ [Ogu79, Sch12]. In a wide sense, the $X(p)$ are the most special K3 surfaces. The uniqueness of $X(p)$ in particular shows that $X(p) \simeq \operatorname{Km}\left(E \times_{\mathbb{F}_{p}} E\right)$ for any supersingular elliptic curve $E$ over $\mathbb{F}_{p}[\mathrm{Ogu} 79$, Shi75].

\subsection{Lifting of K3 surfaces}

Let $X$ be a K3 surface defined over a perfect field $k$ of positive characteristic $p>0$, and let $R$ be a discrete valuation ring with residue field $k$ and field of fractions $K=\operatorname{Frac}(R)$ of characteristic 0 . We call a proper flat (thus smooth) morphism of schemes $X_{R} \rightarrow \operatorname{Spec} R$ which restricts to $X \rightarrow \operatorname{Spec}(k)$ a characteristic 0 model of $X / k$. The generic fiber $X_{K}=X_{R} \otimes_{R} K$ is a K3 surface. For any field $L$ containing $K$, the K3 surface $X_{L}=X_{K} \otimes_{K} L$ is called a lift of $X$ over $L$. Characteristic 0 lifts of $X$ are lifts over some $L$ as above. They are unobstructed [Del81].

\subsection{Geometric lift of an automorphism of a K3 surface}

(See [EO15, Section 2.4].) Let $X$ be a K3 surface defined over a perfect field $k$ of positive characteristic $p>0$, and let $X_{R} \rightarrow$ Spec $R$ be a characteristic 0 model. Recall [BGI71, Chapter X, Appendix] that one has a specialization homomorphism $\mathrm{sp:} \operatorname{Pic}\left(X_{\bar{K}}\right) \rightarrow \operatorname{Pic}(X)$ on the Picard group, which is defined by spreading out and restriction. It is injective as detected in $\ell$-adic cohomology, on which the specialization is an isomorphism [Del77, Chapter V, Theorem 3.1].

One has a restriction homomorphism $\operatorname{Aut}\left(X_{R} / R\right) \rightarrow \operatorname{Aut}(X)$. One defines the subgroup $\operatorname{Aut}^{e}\left(X_{\bar{K}} / \bar{K}\right) \subset \operatorname{Aut}\left(X_{\bar{K}} / \bar{K}\right)$ consisting of those automorphisms which lift to some model $X_{R} \rightarrow \operatorname{Spec} R$. (Here $e$ stands for extendable.) The group law is defined by base change and the composition of automorphisms. Then the restriction homomorphism yields a specialization homomorphism $\iota: \operatorname{Aut}^{e}\left(X_{\bar{K}} / \bar{K}\right) \rightarrow \operatorname{Aut}(X / k)$. Moreover, sp is equivariant under $\iota$. In addition, as automorphisms are detected on the associated formal scheme and $H^{0}\left(X, T_{X / k}\right)=0$, the specialization homomorphism $\iota$ is injective (see [LM11, Lemma 2.3]).

An automorphism $f$ in $\operatorname{Aut}(X)$ is geometrically liftable to characteristic 0 if it is in the image of the specialization homomorphism $\iota$ for some model $X_{R} / R$. 
Theorem 2.1 (See [EO15, Proof of Theorem 6.4]). Let $X$ be a supersingular K3 surface, and let $f \in \operatorname{Aut}(X)$. Assume that the characteristic polynomial $f^{*} \mid \mathrm{NS}(X)$ is irreducible in $\mathbb{Z}[t]$. Then $f$ is never geometrically liftable to characteristic 0 .

Proof. If $f$ lifted geometrically to characteristic 0 , say to $g \in \operatorname{Aut}\left(X_{\bar{K}}\right)$ under $X_{R} \rightarrow \operatorname{Spec} R$, then, as explained above, the specialization map $\iota: \operatorname{NS}\left(X_{\bar{K}}\right) \hookrightarrow \mathrm{NS}(X)$ would be equivariant with respect to $g^{*}$ and $f^{*}$. In particular, the minimal monic polynomial $m_{g}(t) \in \mathbb{Z}[t]$ of $g^{*} \mid \operatorname{NS}\left(X_{\bar{K}}\right)$, which has degree at most 20 , would divide the minimal monic polynomial $m_{f}(t) \in \mathbb{Z}[t]$ of $f^{*} \mid \mathrm{NS}(X)$ in $\mathbb{Z}[t]$, which is irreducible and of degree 22 by assumption. This gives a contradiction.

\section{Preliminaries on Salem numbers and entropy}

In this section, we recall the definition of entropy and Salem numbers, again from [EO15] and the references therein.

In what follows, $L=\left(\mathbb{Z}^{1+t},(*, * *)\right)$ is a hyperbolic lattice, that is, a pair consisting of a free $\mathbb{Z}$-module of rank $1+t$ and a $\mathbb{Z}$-valued symmetric bilinear form $($,$) of \mathbb{Z}^{1+t}$ of signature $(1, t)$ with $t>0$. For any ring $K$, one denotes the scalar extension of $L$ to $K$ by $L_{K}$.

We denote by $\mathrm{O}(L)$ the orthogonal group of the quadratic lattice $L$. It is an algebraic group defined over $\mathbb{Z}$. The determinant

$$
\operatorname{det}: \mathrm{O}(L) \rightarrow\{ \pm 1\}
$$

is a surjective homomorphism of algebraic groups. Its kernel $\mathrm{SO}(L) \subset \mathrm{O}(L)$ is a closed index 2 algebraic subgroup and is the identity component of $\mathrm{O}(L)$. As an algebraic group, $\mathrm{SO}(L)$ is geometrically connected. Moreover, $\mathrm{SO}(L)(\mathbb{R})$ is a connected real Lie group and has index 2 in the real Lie group $\mathrm{O}(L)(\mathbb{R})$, thus is its identity component in the real topology.

The subspace

$$
P:=\left\{x \in L_{\mathbb{R}} \mid\left(x^{2}\right)>0\right\} \subset L_{\mathbb{R}}
$$

consists of two connected components $\pm C$ in the real topology. By continuity, for $g \in \mathrm{O}(L)(\mathbb{R})$, one has $g(C)=C$ or $g(C)=-C$, where the second case also occurs. Thus

$$
\mathrm{O}^{+}\left(L_{\mathbb{R}}\right):=\{g \in \mathrm{O}(L)(\mathbb{R}) \mid g(C)=C\}
$$

is an index 2 closed subgroup of the real Lie group $\mathrm{O}(L)(\mathbb{R})$. Thus $\mathrm{O}^{+}\left(L_{\mathbb{R}}\right)$ is a real Lie subgroup of $\mathrm{O}(L)(\mathbb{R})$, which is not the subgroup of $\mathbb{R}$-points of an algebraic subgroup of $\mathrm{O}(L)$.

One defines

$$
\begin{aligned}
\mathrm{O}^{+}(L) & :=\mathrm{O}(L)(\mathbb{Z}) \cap \mathrm{O}^{+}\left(L_{\mathbb{R}}\right), \\
\mathrm{SO}^{+}(L) & :=\mathrm{O}(L)(\mathbb{Z}) \cap \mathrm{O}^{+}\left(L_{\mathbb{R}}\right) \cap \mathrm{SO}(L)(\mathbb{R}) .
\end{aligned}
$$

The groups $\mathrm{O}^{+}(L)$ and $\mathrm{SO}^{+}(L)$ are abstract subgroups of $\mathrm{O}(L)(\mathbb{Z})$ of index at most 4 .

For application to K3 surfaces, we take $L$ to be the Néron-Severi lattice $\operatorname{NS}(X)$ and $C$ to be the connected component of $P$ containing the ample cone. Thus $\operatorname{Aut}(X)^{*}$, the representation of $\operatorname{Aut}(X)$ on $\mathrm{NS}(X)$, lies in $\mathrm{O}^{+}(\mathrm{NS}(X))$. Moreover, $\operatorname{Aut}(X)^{* 0}:=\operatorname{Aut}(X)^{*} \cap \mathrm{SO}^{+}(\mathrm{NS}(X))$ is a subgroup of $\operatorname{Aut}^{*}(X)$ of index at most 2 .

We call a polynomial $P(x) \in \mathbb{Z}[x]$ a Salem polynomial if it is irreducible, monic, and of even degree $2 d \geqslant 2$, and the complex zeroes of $P(x)$ consist of two real zeroes $a_{1}$ and $a_{2}$ and $d-1$ 


\section{H. Esnault, K. Oguiso and X. Yu}

pairs of complex conjugate zeroes $\alpha_{i}, \bar{\alpha}_{i}(1 \leqslant i \leqslant d-1)$ satisfying

$$
0<a_{2}=\frac{1}{a_{1}}<1, \quad \alpha_{i}, \bar{\alpha}_{i} \in S^{1}:=\{z \in \mathbb{C}|| z \mid=1\} \backslash\{ \pm 1\} .
$$

Proposition 3.1. Let $f \in \mathrm{O}^{+}(L)$. Then, the characteristic polynomial of $f$ is the product of cyclotomic polynomials and at most one Salem polynomial counted with multiplicities.

Proof. As mentioned in [EO15, Proposition 3.1], this is well known. See [McM02, Ogu10].

Definition 3.2. (i) For $f$ as in Proposition 3.1, we define the entropy $h(f)$ of $f$ by

$$
h(f)=\log (r(f)) \geqslant 0,
$$

where $r(f)$ is the spectral radius of $f$, that is, the maximum of the absolute values of the complex eigenvalues of $f$ acting on $L$.

(ii) For a smooth projective surface $S$ and an automorphism $f$ on it, we define the entropy $h(f)$ by

$$
h(f)=\log r\left(f^{*} \mid \mathrm{NS}(S)\right),
$$

where $f^{*}$ is the action on $\mathrm{NS}(S)$ induced by $f$.

This definition is consistent with the topological entropy of automorphisms of smooth complex projective surfaces [ES13].

\section{Two observations from group theory}

In this section, we shall prove Theorem 4.1, relying on [BdlH04] and [BC13], and Theorem 4.6, relying on [Ogu08]. They are crucial for our main results, Theorems 1.2 and 1.1.

ThEOREM 4.1. Let $L$ be a hyperbolic lattice of even rank $2 d$, and let $G \subset \mathrm{SO}^{+}(L)$ be a subgroup. Assume that $G$ has no $G$-stable $\mathbb{R}$-linear subspace of $L_{\mathbb{R}}$ other than $\{0\}$ and $L_{\mathbb{R}}$. Then there is an element $g \in G$, the characteristic polynomial of which is a Salem polynomial of degree $2 d$.

The proof below mimics that of [BC13, Proposition 3.1], where the authors handle the case of $L=\mathrm{NS}(X(2))$, and deduces the statement from [BdlH04, Proposition 1]. We slightly clarify their argument to make it fit with Theorem 4.1.

Proof. Recall [BdlH04, Proposition 1], in which neither the evenness of the rank of $L$ nor the inclusion $G \subset \mathrm{SO}^{+}(L)$ are necessary assumptions.

Theorem 4.2. Let $G \subset \mathrm{O}(L)(\mathbb{R})$ be an abstract subgroup. Assume that $G$ has no $G$-stable $\mathbb{R}$-linear subspace of $L_{\mathbb{R}}$ other than $\{0\}$ and $L_{\mathbb{R}}$. Then the Zariski closure of $G$ in $\mathrm{O}\left(L_{\mathbb{R}}\right)$ contains $\mathrm{SO}\left(L_{\mathbb{R}}\right)$. In particular, if in addition $G \subset \mathrm{SO}(L)(\mathbb{R})$, then its Zariksi closure in $\mathrm{SO}\left(L_{\mathbb{R}}\right)$ is $\mathrm{SO}\left(L_{\mathbb{R}}\right)$.

As is well known, if $L$ is any non-degenerate quadratic lattice and $g \in \mathrm{SO}(L)(\mathbb{Z})$, then if the rank of $L$ is odd, 1 is an eigenvalue of $g$. Indeed, if $Q$ (respectively, $M$ ) is the matrix of the quadratic form of the lattice $L$ (respectively, $g$ ) in a chosen basis, then ${ }^{t} M Q(M-\mathrm{Id})=$ $\left(\mathrm{Id}-{ }^{t} M\right) Q$, thus $\operatorname{det}(M-\mathrm{Id})=-\operatorname{det}(M-\mathrm{Id}) \in \mathbb{Z}$. We now use that in Theorem 4.1, the rank of $L$ is even.

Proposition 4.3. There is an element $g \in \mathrm{SO}(L)(\mathbb{R})$ such that no eigenvalue of $g$ is a root of unity. 


\section{SALEM NUMBERS OF MAXIMAL DEGREE}

Proof. We may choose a real basis

$$
\left\langle v_{1}, v_{2}, v_{3}, \ldots, v_{2 d}\right\rangle
$$

of $L_{\mathbb{R}}$ under which the bilinear form $(*, * *)$ is represented by the matrix $Q:=(1,-1,-1, \ldots,-1)$. We identify $\mathbb{R}$-linear maps and $2 d \times 2 d$-matrices with real entries via this basis. Consider the $\mathbb{R}$-linear map of $L_{\mathbb{R}}$ given by the matrix

$$
M=P \oplus R_{2} \oplus \cdots \oplus R_{d}
$$

where

$$
P=\left(\begin{array}{cc}
\sqrt{2} & 1 \\
1 & \sqrt{2}
\end{array}\right), \quad R_{i}=\left(\begin{array}{cc}
\cos 2 \pi \sqrt{2} & -\sin 2 \pi \sqrt{2} \\
\sin 2 \pi \sqrt{2} & \cos 2 \pi \sqrt{2}
\end{array}\right)
$$

for all $2 \leqslant i \leqslant d$. Then ${ }^{t} M Q M=Q$, $\operatorname{det} M=1$, and the complex eigenvalues of $M$ are

$$
\sqrt{2} \pm 1, \quad e^{ \pm 2 \pi \sqrt{-1} \cdot \sqrt{2}}=\cos (2 \pi \sqrt{2}) \pm \sqrt{-1} \sin (2 \pi \sqrt{2}) .
$$

Note that $e^{ \pm 2 \pi \sqrt{-1} \cdot s}(s \in \mathbb{R})$ is a root of unity if and only if $s$ is rational. Since $\sqrt{2}$ is an irrational real number, it follows that no eigenvalue of $M$ is a root of unity. Thus $M$ satisfies all the requirements.

Now assume $G \subset \mathrm{SO}^{+}(L)$. The argument now closely follows the proof of [BC13, Proposition 3.1].

Lemma 4.4. There are finitely many cyclotomic polynomials of degree at most $2 d$.

Proof. This is because the number of complex numbers with $x^{(2 d) !}=1$ is at most $(2 d)$ !.

Let $P_{2 d} \subset \mathbb{Z}[t]$ be the set of monic polynomials of degree $2 d$. Then $P_{2 d}$ is identified with the affine variety $\mathbb{A}^{2 d}$ defined over $\mathbb{Z}$. The map

$$
\text { char: } \mathrm{SO}(L) \rightarrow P_{2 d}, \quad h \mapsto \Phi_{h}(t):=\operatorname{det}\left(t I_{2 d}-h\right)
$$

is a morphism of affine varieties. Let

$$
u_{1}(t)=t-1, u_{2}(t):=t+1, \ldots, u_{N}(t)
$$

be the cyclotomic polynomials in $\mathbb{Z}[t]$ of degree at most $2 d$, where $N$ is the cardinality of the cyclotomic polynomials of degree at most $2 d$ (Lemma 4.4). The subsets

$$
P_{i}:=\left\{p(t) \in P_{2 d}(\mathbb{C})\left|u_{i}(t)\right| p(t)\right\}
$$

define proper closed algebraic subvarieties of $P_{2 d} \otimes_{\mathbb{Z}} \mathbb{Q}$, so their finite union

$$
Q_{2 d}:=\cup_{i=1}^{N} P_{i} \subset P_{2 d} \otimes_{\mathbb{Z}} \mathbb{Q}
$$

is also one.

Let $g \in G$. Its characteristic polynomial $\Phi_{g}(t) \in \mathbb{Z}[t]$ is monic and of degree $2 d$. By Proposition 3.1, the polynomial $\Phi_{g}(t)$ is the product of cyclotomic polynomials and at most one Salem polynomial, counted with multiplicities. Thus, $\Phi_{g}(t)$ is a Salem polynomial of degree $2 d$ if and only if $\Phi_{g}(t)$ is irreducible and is not a cyclotomic polynomial of degree $2 d$, which is equivalent to saying that no $u_{i}(t)$ divides $\Phi_{g}(t)$ in $\mathbb{Z}[t]$. Since $\Phi_{g}(t)$ and $u_{i}(t)$ are monic polynomials in $\mathbb{Z}[t]$, it follows that no $u_{i}(t)$ divides $\Phi_{g}(t)$ in $\mathbb{Z}[t]$ if and only if no $u_{i}(t)$ divides $\Phi_{g}(t)$ in $\mathbb{C}[t]$. The last condition is, by definition, equivalent to $\Phi_{g}(t) \in P_{2 d}(\mathbb{C}) \backslash Q_{2 d}$. The following lemma completes the proof. 


\section{H. Esnault, K. Oguiso and X. Yu}

Lemma 4.5. There is an element $g \in G$ such that $\Phi_{g}(t) \in P_{2 d}(\mathbb{C}) \backslash Q_{2 d}$.

Proof. By our assumption, we can apply Theorem 4.2 to $G$, so the Zariski closure of $G$ in $\operatorname{SO}\left(L_{\mathbb{R}}\right)$ is $\mathrm{SO}\left(L_{\mathbb{R}}\right)$. On the other hand, $\operatorname{char}^{-1}\left(P_{2 d} \otimes_{\mathbb{Z}} \mathbb{Q} \backslash Q_{2 d}\right)$ is Zariski open in $\mathrm{SO}(L) \otimes_{\mathbb{Z}} \mathbb{Q}$, and not empty by Proposition 4.3. Thus it intersects $G \subset \mathrm{SO}(L)(\mathbb{Q})$ non-trivially. This finishes the proof.

This completes the proof of Theorem 4.1.

The following theorem is deduced from [Ogu08, Proof of Lemma 3.6, Claim 3.8].

TheOREM 4.6. Let $L$ be a hyperbolic lattice of signature $(1, r+1)$ with $r \geqslant 0$, and let $e \in L$ be a primitive element such that $(e, e)=0$. Let $G \subset \mathrm{SO}(L)(\mathbb{Z})$ such that $G \simeq \mathbb{Z}^{r}$ as a group and such that $g(e)=e$ for all $g \in G$. Then any $G$-stable $\mathbb{R}$-linear subspace $M$ of $L_{\mathbb{R}}$ either is in the hypersurface $e^{\perp}$ in $L_{\mathbb{R}}$ or satisfies $M=L_{\mathbb{R}}$.

Proof. Note that $G \subset \mathrm{SO}^{+}(L)$. Indeed, for $g \in G$, we have $g(e)=e$. Any small enough open ball in $L_{\mathbb{R}}$ in the classical topology, centered in $e$, meets $C$ in an open $\mathcal{U}$ such that for any $x \in \mathcal{U}$, the distance between $g(x)$ and $x$ is small enough that it forces $g(x)$ to lie in $C$.

Lemma 4.7. There are an integral basis

$$
\left\langle e, w_{1}, \ldots, w_{r}\right\rangle
$$

of $e^{\perp} \subset L$, so necessarily $\left(w_{j}, w_{j}\right)<0$ for all $1 \leqslant j \leqslant r$, a $\mathbb{Q}$-basis

$$
\left\langle e, w_{1}, \ldots, w_{r}, u\right\rangle
$$

of $L_{\mathbb{Q}}$ with $(e, u)=1$, and a finite-index subgroup

$$
H:=\left\langle g_{1}, \ldots, g_{r}\right\rangle \simeq \mathbb{Z}^{r}
$$

of $G$ such that

$$
g_{i}=\left(\begin{array}{ccc}
1 & \mathbf{a}_{i}^{t} & c_{i} \\
\mathbf{0} & I_{r} & q_{i} \mathbf{e}_{i} \\
0 & \mathbf{0}^{t} & 1
\end{array}\right)
$$

under the $\mathbb{Q}$-basis above. Here $1,0 \in \mathbb{Q}$ are the unit and the zero, respectively, $c_{i}$ and $q_{i} \neq 0$ are in $\mathbb{Q}$, and $\mathbf{e}_{i}$ is the ith unit vector of $\mathbb{Q}^{r}$. Furthermore, $I_{r}$ is the $r \times r$ identity matrix, $\mathbf{0} \in \mathbb{Q}^{r}$ is the zero vector, $\mathbf{a}_{i}^{t}$ is the transpose of a column vector $\mathbf{a}_{i} \in \mathbb{Q}^{r}$, and similarly for $\mathbf{0}^{t}$.

Proof. This is observed in [Ogu08, Proof of Lemma 3.6, Claim 3.8]. The essential part is that $e^{\perp} / \mathbb{Z} e$ is a negative-definite lattice with respect to the $\mathbb{Z}$-valued bilinear form induced by $(*, * *)$ and $G$ acts on this negative-definite lattice $e^{\perp} / \mathbb{Z} e$. Note that

$$
H:=\operatorname{Ker}\left(G \rightarrow \mathrm{O}\left(e^{\perp} / \mathbb{Z} e\right)(\mathbb{Z})\right)
$$

is a finite-index subgroup of $G$, as $\mathrm{O}\left(e^{\perp} / \mathbb{Z} e\right)(\mathbb{Z})$ is a finite group. Since $G \simeq \mathbb{Z}^{r}$, we then have $H \simeq \mathbb{Z}^{r}$ by the fundamental theorem of finitely generated abelian groups. Next, choose a $\mathbb{Z}$-basis

$$
\left\langle e, w_{1}, \ldots, w_{r}\right\rangle
$$

of $e^{\perp}$. As $e^{\perp}$ is of signature $(0,1, r-1)$ (degenerate lattice), it follows that $\left(w_{i}, w_{i}\right)<0$ and $\left(e . w_{i}\right)=0$, in addition to $\left(e^{2}\right)=0$. Choose $u \in L_{\mathbb{Q}}$ such that $(e . u)=1$. Such a vector $u$ exists as $L$ is hyperbolic. Then,

$$
\left\langle e, w_{1}, \ldots, w_{r}, u\right\rangle
$$


is a $\mathbb{Q}$-basis of $L_{\mathbb{Q}}$ and the matrix representation of $H$ with respect to this $\mathbb{Q}$-basis is of the form

$$
g=\left(\begin{array}{ccc}
1 & \mathbf{a}^{t}(g) & c(g) \\
\mathbf{0} & I_{r} & \mathbf{b}(g) \\
0 & \mathbf{0}^{t} & 1
\end{array}\right)
$$

for all $g \in H \subset G \subset \mathrm{SO}(L)(\mathbb{Z})$. It is proved in [Ogu08, Claim 3.8] that the map

$$
H \rightarrow \mathbb{Q}^{r}, \quad g \mapsto \mathbf{b}(g)
$$

is an injective group homomorphism. This is easily checked by an explicit computation of matrices. Since $H \simeq \mathbb{Z}^{r}$, it follows that

$$
\mathbb{Z}^{r} \simeq H \simeq\langle\mathbf{b}(g) \mid g \in H\rangle \subset \mathbb{Q}^{r} .
$$

Thus, again by the fundamental theorem of finitely generated abelian groups, we obtain a $\mathbb{Z}$-basis of $H$ with the required property.

Lemma 4.8. There are integers $i, j$ such that $1 \leqslant i, j \leqslant r$ and $\mathbf{a}_{j}^{t} \cdot \mathbf{e}_{i} \neq 0$. Here the left-hand side is a product of matrices and we naturally identify $1 \times 1$ matrices with their entry.

Proof. Assume to the contrary that $\mathbf{a}_{j}^{t} \cdot \mathbf{e}_{i}=0$ for all $i, j$. Then $\mathbf{a}_{j}^{t}=0$ for all $j$. Thus

$$
g_{j}(u)=c_{j} e+q_{j} w_{j}+u
$$

by the explicit matrix form. Then by induction one has

$$
g_{j}^{k}(u)=g_{j}\left((k-1) c_{j} e+(k-1) w_{j}+u\right)=k\left(c_{j} e+q_{j} w_{j}\right)+u
$$

for all positive integer $k$. Since $g_{j}^{k}$ preserves the intersection form, it follows that

$$
(u, u)=\left(g^{k}(u), g^{k}(u)\right)=(u, u)+2 k\left(q_{j}\left(w_{j}, u\right)+c_{j}\right)+k^{2} q_{j}^{2}\left(w_{j}, w_{j}\right),
$$

whence

$$
\left(w_{j}, w_{j}\right) q_{j}^{2} k+2\left(c_{j}+q_{j}\left(w_{j}, u\right)\right)=0
$$

for all positive integers $k$, which contradicts the inequalities $\left(w_{j}, w_{j}\right)<0$ and $q_{j} \neq 0$ in Lemma 4.7.

To conclude the proof of Theorem 4.6, it suffices to confirm the following.

Lemma 4.9. Let $M$ be a $G$-stable $\mathbb{R}$-linear subspace of $L_{\mathbb{R}}$. Assume that there is a $v \in M_{\mathbb{R}}$ such that $v \notin e^{\perp}$ in $L_{\mathbb{R}}$. Then, $M=L_{\mathbb{R}}$.

Proof. By replacing $v$ by a multiple by $\mathbb{R}^{\times}$, we may assume without loss of generality that

$$
v=x e+\sum_{s=1}^{r} y_{s} w_{s}+u,
$$

where $x$ and $y_{s}$ are real numbers. Then, by Lemma 4.7,

$$
g_{i}(v)=v+\left(\mathbf{a}_{i}^{t} \cdot \mathbf{y}+c_{i}\right) e+q_{i} w_{i}
$$

where $\mathbf{y} \in \mathbb{R}^{r}$ is the vector whose $s$ th entry is $y_{s}$. But $g(v) \in M$ by assumption, and by the fact that $M$ is $\mathbb{R}$-linear it follows that

$$
v_{i}:=\left(\mathbf{a}_{i}^{t} \cdot \mathbf{y}+c_{i}\right) e+q_{i} w_{i} \in M .
$$




\section{H. Esnault, K. Oguiso and X. Yu}

By Lemma 4.7, it follows that

$$
g_{j}\left(v_{i}\right)=v_{i}+q_{i}\left({ }^{t} \mathbf{a}_{j} \cdot \mathbf{e}_{i}\right) e .
$$

Recall from Lemma 4.7 that $q_{i} \neq 0$. Then, for the same reason as above, it follows that

$$
\left(\mathbf{a}_{j}^{t} \cdot \mathbf{e}_{i}\right) e \in M
$$

for all $1 \leqslant i, j \leqslant r$. Thus by Lemma 4.8, we have $e \in M$. Combining this with $v_{i} \in M$, it follows that $q_{i} w_{i} \in M$ for all $i$. Since $q_{i} \neq 0$, it follows that $w_{i} \in M$ for all $i$. Combining this with $v \in M$, it follows that $u \in M$. Since $e, w_{i}, u$ form a $\mathbb{Q}$-basis of $L_{\mathbb{Q}}$, thus an $\mathbb{R}$-basis of $L_{\mathbb{R}}$, it follows that $L_{\mathbb{R}} \subset M$. This finishes the proof.

This completes the proof of Theorem 4.6.

\section{Proof of Theorems 1.2 and 1.1}

First we prove Theorem 1.2. Let $X$ be as in Theorem 1.2. We recall that $\mathrm{NS}(X)$ is a hyperbolic lattice of signature $(1, \rho(X)-1)$.

Recall that an elliptic fibration $\varphi: X \rightarrow \mathbb{P}^{1}$ on the minimal surface $X$ is a projective, surjective morphism over a field $k$, which has a section $O: \mathbb{P}^{1} \rightarrow X$, and is such that the generic fiber is a smooth curve of genus 1 over $k\left(\mathbb{P}^{1}\right)$. We denote by $\operatorname{MW}(\varphi)$ its Mordell-Weil group, viewed as a subgroup of the group of the birational automorphisms $\operatorname{Bir}(X)$.

The following lemma is well known.

Lemma 5.1. Let $\varphi: X \rightarrow \mathbb{P}^{1}$ be an elliptic fibration. Then

- the Mordell-Weil group $\operatorname{MW}(\varphi)$ of $\varphi$ is a finitely generated abelian subgroup of $\operatorname{Aut}(X) \subset$ $\operatorname{Bir}(X)$;

- the action of $\operatorname{MW}(\varphi)$ on $\mathrm{NS}(X)$ is faithful.

We denote by $\mathrm{MW}(\varphi)^{*} \subset \mathrm{O}^{+}(\mathrm{NS}(X))$ the image of the induced representation on the NéronSeveri group $\mathrm{NS}(X)$ of $X$. Thus $\mathrm{MW}(\varphi) \stackrel{\cong}{\rightarrow} \operatorname{MW}(\varphi)^{*}$.

Proof. The group $\mathrm{MW}(\varphi)$ is finitely generated by [Shi90], as $\varphi$, for topological reasons, has at least one singular fiber. As $X$ is a smooth projective minimal surface, $\operatorname{Bir}(X)=\operatorname{Aut}(X)$. This implies part (i). One has $|O|=\{O\}$, where $|O|$ is the complete linear system containing $O$. This is because $O \simeq \mathbb{P}^{1}$ and $(O, O)=-2<0$, as $X$ is a $\mathrm{K} 3$ surface.

Let $f \in \operatorname{MW}(\varphi)$. If $f^{*} \mid \mathrm{NS}(X)=\mathrm{Id}$, then, in particular, the class of $O$ in $\mathrm{NS}(X)$ is invariant under $f^{*}$. Thus $|O|$ is invariant under $f$, and therefore the section $O$ is invariant under $f$. As $f \in \operatorname{MW}(\varphi)$, this implies $f=\operatorname{Id}$ on $X$. This proves part (ii).

From here on, the notation is as in Section 3. In addition, we define

$$
\operatorname{MW}(\varphi)^{* 0}:=\operatorname{MW}(\varphi)^{*} \cap \mathrm{SO}^{+}\left(\mathrm{NS}(X)_{\mathbb{R}}\right) .
$$

Lemma 5.2. (i) The group $\operatorname{Aut}(X)^{* 0}$ is infinite; thus $\operatorname{Aut}(X)$ is infinite as well.

(ii) The abelian groups $\operatorname{MW}(\varphi)$ and $\operatorname{MW}(\varphi)^{* 0}$ have the same rank.

Proof. As $\operatorname{Aut}(X)^{* 0} \supset \mathrm{MW}(\varphi)^{* 0}$, statement (i) follows from statement (ii). We prove statement (ii). By Lemma 5.1, we have $\mathrm{MW}(\varphi) \stackrel{\cong}{\rightarrow} \mathrm{MW}(\varphi)^{*}$. On the other hand, $\mathrm{SO}^{+}\left(\mathrm{NS}(X)_{\mathbb{R}}\right)$ is a finite-index subgroup of $\mathrm{O}(\mathrm{NS}(X))(\mathbb{R})$. Thus $\mathrm{MW}(\varphi)^{* 0}$ is a finite-index subgroup of $\mathrm{MW}(\varphi)^{*}$. 
Recall that $X$ is as in Theorem 1.2. We denote by $e_{1} \in \mathrm{NS}(X)$ the class of a fiber of $\varphi_{1}$. Lemma 5.3. The surface $X$ admits a $g \in \operatorname{Aut}(X)$ such that $g^{*}\left(e_{1}\right) \neq e_{1}$ in $\operatorname{NS}(X)$.

Proof. By the assumption, $X$ admits a different elliptic fibration $\varphi_{2}: X \rightarrow \mathbb{P}^{1}$ of positive MordellWeil rank. Thus, by Lemma 5.2, there is a $g \in \operatorname{MW}\left(\varphi_{2}\right)$ of infinite order.

Let $f \in \mathrm{NS}(X)$ be the fiber class of $\varphi_{2}$; thus, in particular, $g^{*}(f)=f$. By the Hodge index theorem, $\left(f+e_{1}, f+e_{1}\right)>0$. Therefore, the lattice $\left(e_{1}+f\right)^{\perp}$ in $\mathrm{NS}(X)$ is negative definite.

Assume $g^{*}\left(e_{1}\right)=e_{1}$ in $\operatorname{NS}(X)$. Then $g^{*}\left(e_{1}+f\right)=e_{1}+f$. Thus, by Lemma 5.1 , the morphism $g$ acts faithfully on $\left(e_{1}+f\right)^{\perp}$. As $\mathrm{O}\left(\left(e_{1}+f\right)^{\perp}\right)(\mathbb{Z})$ is a finite group, $g$ is of finite order, which gives a contradiction. This proves the lemma.

Definition 5.4. Consider all the elliptic fibrations $\Phi_{i}: X \rightarrow \mathbb{P}^{1}(i \in I)$ on $X$ with maximum Mordell-Weil rank $r=\rho(X)-2$. Let $e_{i} \in \operatorname{NS}(X)$ be the class of fibers of $\Phi_{i}$. Set

$$
\mathcal{S}:=\left\{e_{i} \in \mathrm{NS}(X) \mid i \in I\right\},
$$

and denote by $\mathbb{R}\langle\mathcal{S}\rangle \subset \mathrm{NS}(X)_{\mathbb{R}}$ the real linear subspace spanned by $\mathcal{S}$. Note that $\left(e_{i}, e_{i}\right)=0$, $e_{i}$ is numerically effective, and $e_{i}$ is primitive in $\operatorname{NS}(X)$ for all $e_{i} \in \mathcal{S}$.

LEMmA 5.5. One has $\mathbb{R}\langle\mathcal{S}\rangle \stackrel{\cong}{\rightrightarrows} \mathrm{NS}(X)_{\mathbb{R}}$.

Proof. Recall that $\mathcal{S} \neq \emptyset$ by our assumption. Let $e_{1} \in \mathcal{S}$ be the class of $\Phi_{1}$. By definition of $\mathcal{S}$, $\mathbb{R}\langle\mathcal{S}\rangle$ is Aut $(X)$-stable. Let $g \in \operatorname{Aut}(X)$ with $e_{2}:=g\left(e_{1}\right) \neq e_{1}$ (Lemma 5.3). As $\mathcal{S}$ is stable under $\operatorname{Aut}(X)$, it follows that $e_{2} \in \mathcal{S}$ as well.

Assume to the contrary that $\mathbb{R}\langle\mathcal{S}\rangle \neq \mathrm{NS}(X)_{\mathbb{R}}$. Let $G_{1} \subset \mathrm{MW}\left(\Phi_{1}\right)^{* 0}$ be a free abelian group of rank $r=\rho(X)-2$. By Theorem 4.6 applied to $G_{1}$, one has $\mathbb{R}\langle\mathcal{S}\rangle \subset e_{1}^{\perp}$ in $\operatorname{NS}(X)_{\mathbb{R}}$. By the Hodge index theorem and the fact that the $e_{i}$ are primitive, one has $\left(e_{1}, e_{2}\right)>0$, which gives a contradiction.

Lemma 5.6. There is no Aut $(X)^{* 0}$-stable $\mathbb{R}$-linear subspace of $\mathrm{NS}(X)_{\mathbb{R}}$ other than $\{0\}$ and $\mathrm{NS}(X)_{\mathbb{R}}$

Proof. Let $M \neq \mathrm{NS}(X)_{\mathbb{R}}$ be an $\operatorname{Aut}(X)^{* 0}$-stable $\mathbb{R}$-linear subspace of $\mathrm{NS}(X)_{\mathbb{R}}$. For $\Phi_{i}$ as in Definition 5.4, let $G_{i} \subset \mathrm{MW}\left(\Phi_{i}\right)^{* 0}$ be a free abelian subgroup of maximal rank $r=\rho(X)-2$ (Lemma 5.1). By Theorem 4.6 applied to $G_{i}$, it follows that

$$
M \subset \cap_{i \in I} e_{i}^{\perp} \subset \mathrm{NS}(X)_{\mathbb{R}}
$$

As the vectors $e_{i}$, for $i \in I$, generate $\mathrm{NS}(X)_{\mathbb{R}}$ and the intersection form is non-degenerate on $\mathrm{NS}(X)$, it follows that

$$
\cap_{i \in I} e_{i}^{\perp}=\{0\}
$$

This proves the lemma.

Proof of Theorem 1.2. By Lemmas 5.2 and 5.6, we can apply Theorem 4.1.

Proof of Theorem 1.1. As mentioned in the introduction, Shioda [Shi13] proved that any $X(p)$ with $p=11$ or $p>13$ admits an elliptic fibration $\varphi_{1}: X \rightarrow \mathbb{P}^{1}$ with Mordell-Weil rank $20=22-2$. On the other hand, over $\mathbb{F}_{p}$, one has $X(p) \simeq \operatorname{Km}\left(E \times_{\mathbb{F}_{p}} E\right)$ for a supersingular elliptic curve $E$ over $\mathbb{F}_{p}$, and the fibration $\varphi_{2}: X \rightarrow \mathbb{P}^{1}$ induced by the first projection $E \times_{\mathbb{F}_{p}} E \rightarrow E$ is an elliptic fibration with Mordell-Weil rank 4 (thus positive) over $\overline{\mathbb{F}}_{p}$, by the formula for the Mordell-Weil rank [Shi90]. In particular, these two fibrations are non-isomorphic. So, we apply Theorem 1.2 to deduce Theorem 1.1 for $p=11$ or $p>13$. The cases $p=2$ and $p=3$ are proved by [BC13] and [EO15]. This completes the proof of Theorem 1.1. 


\section{H. Esnault, K. Oguiso and X. Yu}

\section{ACKNOWLEDGEMENTS}

We thank S. Cantat, B. Gross, and C. T. McMullen for discussions. The first author thanks the Department of Mathematics of Harvard University for hospitality, the third author thanks T. Hibi for financial support during his stay at Osaka University.

\section{REFERENCES}

Art74 M. Artin, Supersingular K3 surfaces, Ann. Sci. École Norm. Sup. (4) 7 (1974), no. 4, 543-567; http://www . numdam. org/item?id=ASENS_1974_4_7_4_543_0.

BC13 L. Blanc and S. Cantat, Dynamical degrees of birational transformations of projective surfaces, 2013, arXiv:1307.0361.

BGI71 P. Berthelot, A. Grothendieck, and L. Illusie, Théorie des intersections et théorème de RiemannRoch (Séminaire de Géométrie Algébrique du Bois-Marie 1966-1967 (SGA 6)), Lecture Notes in Math., vol. 225 (Springer-Verlag, Berlin - New York, 1971); http://dx.doi.org/10.1007/ $\mathrm{BFb0066283.}$

BdlH04 Y. Benoist and P. de la Harpe, Adhérence de Zariski des groupes de Coxeter, Compos. Math. 140 (2004), no. 5, 1357-1366; http://dx.doi.org/10.1112/S0010437X04000338.

Del77 P. Deligne, Cohomologie étale (Séminaire de Géométrie Algébrique du Bois-Marie (SGA 4 $\frac{1}{2}$ )), Lecture Notes in Math., vol. 569 (Springer-Verlag, Berlin - New York, 1977), http://dx.doi. org/10.1007/BFb0091516.

Del81_, Relèvement des surfaces K3 en caractéristique nulle, Algebraic Surfaces (Orsay, 197678), Lecture Notes in Math., vol. 868 (Springer, Berlin - New York, 1981), 58-79; http://dx. doi.org/10.1007/BFb0090646.

DK03 I. Dolgachev and S. Kondō, A supersingular K3 surface in characteristic 2 and the Leech lattice, Int. Math. Res. Not. 2003 (2003), no. 1, 1-23; http://dx.doi.org/10.1155/ S1073792803202038.

EO15 H. Esnault and K. Oguiso, Non-liftability of automorphism groups of a K3 surface in positive characteristic, Math. Ann. 363 (2015), no. 3, 1187-1206; http://dx.doi.org/10.1007/ s00208-015-1197-9.

ES13 H. Esnault and V. Srinivas, Algebraic versus topological entropy for surfaces over finite fields, Osaka J. Math. 50 (2013), no. 3, 827-846; http://projecteuclid.org/euclid.ojm/ 1380287436.

GM02 B.H. Gross and C.T. McMullen, Automorphisms of even unimodular lattices and unramified Salem numbers, J. Algebra 257 (2002), no. 2, 265-290; http://dx.doi.org/10.1016/ S0021-8693(02) 00552-5.

Jan14 J. Jang, Representations of the automorphism group of a supersingular K3 surface of Artininvariant 1 over odd characteristic, J. Chungcheong Math. Soc. 27 (2014), no. 2, 287-295; http: //dx.doi.org/10.14403/jcms.2014.27.2.287.

KS14 S. Kondō and I. Shimada, The automorphism group of a supersingular K3 surface with Artin invariant 1 in characteristic 3, Int. Math. Res. Not. 2014 (2014), no. 7, 1885-1924; http: //dx.doi.org/10.1093/imrn/rns274.

LM11 M. Lieblich and D. Maulik, A note on the cone conjecture for K3 surfaces in positive characteristic, 2011, arXiv:1102.3377.

McM02 C. T. McMullen, Dynamics on K3 surfaces: Salem numbers and Siegel disks, J. reine angew. Math. 545 (2002), 201-233; http://dx.doi.org/10.1515/crll.2002.036.

McM16 _ Automorphisms of projective K3 surfaces with minimum entropy, Invent. Math. 203 (2016), no. 1, 179-215; http://dx.doi.org/10.1007/s00222-015-0590-z.

Ogu79 A. Ogus, Supersingular K3 crystals, Astérisque 64 (Soc. Math. France, Paris, 1979), 3-86. 


\section{SALEM NUMBERS OF MAXIMAL DEGREE}

Ogu83 A A crystalline Torelli theorem for supersingular K3 surfaces, Arithmetic and Geometry, Vol. II, Progr. Math., vol. 36 (Birkhäuser Boston, Boston, MA, 1983), 361-394; http://dx. doi. org/10.1007/978-1-4757-9286-7_14.

Ogu08 K. Oguiso, Mordell-Weil groups of a hyperkähler manifold-a question of F. Campana, Publ. Res. Inst. Math. Sci. 44 (2008), no. 2, 495-506; http://dx.doi.org/10.2977/prims/1210167335.

Ogu10_, Salem polynomials and the bimeromorphic automorphism group of a hyper-Kähler manifold, Selected Papers on Analysis and Differential Equations, Amer. Math. Soc. Transl. Ser. 2, vol. 230 (Amer. Math. Soc., Providence, RI, 2010), 201-227.

Sch12 M. Schütt, A note on the supersingular K3 surface of Artin invariant 1, J. Pure Appl. Algebra 216 (2012), no. 6, 1438-1441; http://dx.doi.org/10.1016/j.jpaa.2011.10.036.

Shi75 T. Shioda, Algebraic cycles on certain K3 surfaces in characteristic p, Manifolds - Tokyo 1973 (Proc. Internat. Conf., Tokyo, 1973) (Univ. Tokyo Press, Tokyo, 1975), 357-364.

Shi90_, On the Mordell-Weil lattices, Comment. Math. Univ. St. Paul. 39 (1990), no. 2, 211-240.

Shi13_, Elliptic fibrations of maximal rank on a supersingular K3 surface, Izv. Math. 77 (2013), no. 3, 571-580; http://dx.doi.org/10.1070/IM2013v077n03ABEH002649.

Hélène Esnault esnault@math.fu-berlin.de

Freie Universität Berlin, Arnimallee 3, 14195, Berlin, Germany

Keiji Oguiso oguiso@ms.u-tokyo.ac.jp

Mathematical Sciences, the University of Tokyo, Meguro Komaba 3-8-1, Tokyo, Japan

Korea Institute for Advanced Study, Hoegiro 87, Seoul, 133-722, Korea

Xun Yu yxn100135@postech.ac.kr

Center for Geometry and its Applications, POSTECH, Pohang, 790-784, Korea 